\title{
FASE DE OVO EM Spermologus rufus BOHEMAN (COLEOPTERA: CURCULIONIDAE) ${ }^{1}$
}

\author{
Spermologus rufus BOHEMAN \\ (COLEOPTERA: CURCULIONIDAE) EGG PHASE
}

\author{
Marliton Rocha BARRETO ${ }^{2}$ - \\ Norivaldo dos ANJOS ${ }^{3}$
}

\begin{abstract}
RESUMO
Caracterizou-se a fase de ovo Spermologus rufus Boheman 1843 (Coleoptera: Curculionidae), em condições laboratoriais $\left(T=24,9 \pm 0,9^{\circ} \mathrm{C}\right.$; UR $=78,5 \pm 5,9 \%$ e fotofase de 12 horas). Adultos sexados e divididos em casais foram colocados em frascos de Wheaton, contendo cada um três sementes de Araucaria angustifolia. As sementes eram trocadas diariamente caso fossem ovipositadas sendo, então, dissecadas para acompanhar o desenvolvimento dos insetos na fase de ovo. O ovo apresentou coloração branco-amarelada, formato oblongo e córion transparente, membranoso e aparentemente liso. A maior largura média encontrada foi de $0,63 \pm 0,02 \mathrm{~mm}$ e o comprimento médio, de $1,06 \pm 0,03 \mathrm{~mm}$. O período de incubação variou de dois a seis dias e a maior freqüência de eclosão larval ocorreu entre o terceiro e o quarto dia de incubação. A viabilidade média por fêmea foi de $97,91 \pm 1,66 \%$ e a mortalidade média foi de $2,07 \%$.
\end{abstract}

Palavras-chave: Insecta, biologia, oviposição, Araucaria.

\begin{abstract}
To characterize the Spermologus rufus Boheman 1843 (Coleoptera: Curculionidae) egg phase was carried out the study under controlled conditions $\left(T=24.9 \pm 0,9^{\circ} \mathrm{C}\right.$; UR $=78.5 \pm 5,9 \%$ and 12 hours footofase). Pairs of adults (male+female) were placed in Wheaton flasks containing three Araucaria angustifolia seeds each. The seeds were replaced daily when oviposition occurred. Seeds containing eggs were dissected in order to accompany insect development during the egg phase. The eggs are yellowish-white, oblong, membranous, apparently smooth with a transparent corion. The average largest width was $0.63 \pm 0.02 \mathrm{~mm}$ and the average lenght was $1.06 \pm 0.03 \mathrm{~mm}$. The incubation period varied from two to six days and the highest frequency of larval eclosion occurred between the third and the fourth day days of incubation. The mean viability per female was equal to $97.91 \pm 1.66 \%$ and the mean mortality was $2.07 \%$.
\end{abstract}

Key words: Insecta, biology, oviposition, Araucaria.

\footnotetext{
${ }^{1}$ Parte da Dissertação de Mestrado do primeiro autor, apresentada à Universidade Federal de Viçosa, com bolsa da CAPES.

2 Biólogo, Doutorando em Entomologia, Departamento de Zoologia, Universidade Federal do Paraná, Caixa Postal 19020, CEP 81.531-990, Curitiba, PR. =- Autor para correspondência.

${ }^{3}$ Engenheiro Florestal, Doutor, Universidade Federal de Viçosa, CEP 35.570-000, Viçosa, MG.
} 


\section{INTRODUÇÃO}

O pinheiro-do-paraná, Araucaria angustifolia (Bert.) Kuntze ocorre nos Estados do Rio Grande do Sul, Paraná, Santa Catarina, Minas Gerais, São Paulo e Rio de Janeiro [11]. Essa espécie apresenta significativa participação no fornecimento de madeira nobre, empregada nos mais diferentes setores das indústrias madeireira e papeleira [6,11]. Além disso, ostenta rica biocenose formada principalmente por espécies de insetos [6,17], que vivem nas diferentes partes desse vegetal, onde se abrigam e/ou, encontram seu alimento [5]. Segundo Mattos (1972) [12], a principal forma de propagação dessa espécie florestal se dá pela semente.

Dentre as espécies de insetos que danificam sementes de $A$. angustifolia, a Spermologus rufus Boheman (Coleoptera: Curculionidae) foi relatada por Barreto et al. (1996) [2], sendo que até então, esta espécie somente havia sido relatada danificando amêndoas de Theobroma cacao [4]. A distribuição geográfica desse inseto abrange os Estados da Bahia, São Paulo [17] e Minas Gerais [2, 3]. Informações preliminares com relação à morfologia do ovo foram descritas por Bondar (1925) [4]. Entretanto, como essas informações não satisfaziam às necessidades atuais do conhecimento sobre a biologia da espécie, neste trabalho buscouse caracterizar a fase de ovo de S. rufus.

\section{MATERIAL E MÉTODOS}

Os trabalhos foram realizados no Laboratório de Entomologia Florestal da Universidade Federal de Viçosa, em Viçosa, MG, em condições controladas $\left(T=24,9 \pm 0,9^{\circ} \mathrm{C} ; \quad U R=78,5 \pm 5,9 \%\right.$ e fotofase de 12 horas). O início da criação deu-se a partir de insetos adultos coletados em sementes de Araucaria angustifolia comercializadas na região de Viçosa, MG.

A criação dos insetos em número suficiente aos propósitos da pesquisa foi feita utilizando-se gaiolas teladas de dimensões $20 \times 20 \times 20 \mathrm{~cm}$. A partir da criação, adultos obtidos foram sexados, divididos em casais e colocados em frascos de Wheaton $(60 \mathrm{ml})$, com tampa de pressão perfurada, contendo cada um três sementes de $A$. angustifolia, que serviram de substrato alimentar e de oviposição. As sementes foram trocadas diariamente caso fossem ovipositadas sendo, então, dissecadas para acompanhar o desenvolvimento do inseto na fase de ovo. As sementes utilizadas nos experimentos apresentaram teor de umidade de $37 \%$, determinado por meio do método-padrão da estufa, utilizando-se temperatura constante de $105^{\circ} \mathrm{C}$ por 24 horas [7].

Para caracterizar a forma e coloração, utilizaram-se ovos provenientes de 20 fêmeas. As dimensões foram obtidas medindo-se o comprimento e a maior largura dos ovos extraídos das sementes dissecadas. A coloração foi determinada por meio de observações diretas. Determinou-se o comprimento e a maior largura de 100 ovos utilizando-se ocular micrométrica adaptada a microscópio estereoscópico.

Por se tratar de inseto que oviposita no interior das sementes, o período de incubação foi determinado considerando-se o tempo mínimo decorrido entre o dia da oviposição e o dia anterior aquele em que se constataram as primeiras larvas e o tempo máximo, aquele decorrido entre o dia da oviposição e o da última constatação de ovos [15]. Os dados foram obtidos a cada 24 horas.

Para a caracterização do comportamento embrionário observou-se a manifestação de qualquer atividade que $o$ embrião pudesse apresentar em função de fatores ambientais e do desenvolvimento. A viabilidade dos ovos foi determinada com base na diferença obtida entre a quantidade total de ovos depositados e o percentual de ovos inviáveis divididos pela soma da quantidade total de ovos, larvas, pupas e adultos constatados nas dissecações das sementes. Foram considerados como ovos inviáveis aqueles em que se constatou a presença de fungo e aqueles colocados em locais que impediram seu desenvolvimento.

\section{RESULTADOS E DISCUSSÃO}

O ovo de $S$. rufus apresentou formato oblongo e córion transparente, membranoso e aparentemente liso, com coloração brancoamarelada, permanecendo assim durante todo o desenvolvimento embrionário (Figura 1). O formato é semelhante ao descrito por Bondar (1925) [4].

A largura média dos ovos foi de $0,63+0,02 \mathrm{~mm}$ (variando de 0,56 a $0,75 \mathrm{~mm}$ ) e o comprimento médio foi de $1,06 \pm 0,03 \mathrm{~mm}$ (variando de 0,80 a $1,18 \mathrm{~mm}$ ) (Tabela 1 ).

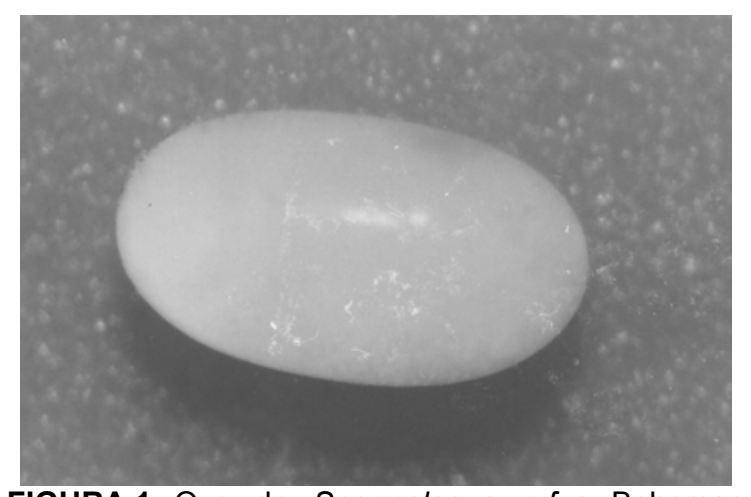

FIGURA 1 -Ovo de Spermologus rufus Boheman (aumento de 75x), Viçosa, MG, 1997

O período de incubação variou de dois a seis dias (Figura 2). A maior freqüência de eclosão larval ocorreu entre o terceiro e o quarto dia de incubação. O período de incubação encontrado em S. rufus foi próximo ao verificado por Bondar (1925) [4] nesta mesma espécie, que apresentou o mínimo de cinco e máximo de oito dias, e para Marshallius bonellii Boheman, que apresentou o mínimo de três dias e o 
máximo de sete dias [10]. As diferenças encontradas no período de incubação podem ser atribuídas às variações de temperatura ou de umidade do ambiente, conforme relataram Moorhouse et al. (1992) [13].

A viabilidade média dos ovos por fêmea foi igual a $97,9+1,6 \%$ (mínima de $91,8 \%$ e máxima de $99,6 \%$ (Tabela 2). A essa alta viabilidade encontrada pode estar associada a qualidade da semente utilizada, pois sabe-se que a qualidade nutricional é fator importante para uma elevada viabilidade dos ovos [18]. Não se observou diminuição na viabilidade dos ovos à medida que as fêmeas fizeram mais posturas e ficaram mais velhas. Essa tendência encontrada em S. rufus é contrária ao observado em Otiorhynchus sulcatus Fabricius, que, segundo Moorhouse et al. (1992) [14], diminuiu a viabilidade dos ovos com o passar dos dias.

TABELA 1 - Comprimento (C) e largura (L), em milímetros, de ovos (5 repetições) de Spermologus rufus Boheman $\left(T=24,9+0,9^{\circ} \mathrm{C}\right.$, UR $=78,5+5,9 \%$ e fotofase $=12$ horas $)$. Viçosa, MG, 1997.

\begin{tabular}{|c|c|c|c|c|c|c|c|c|c|c|c|c|}
\hline \multirow{3}{*}{$\begin{array}{l}\text { Fêmea } \\
\text { Número }\end{array}$} & \multicolumn{12}{|c|}{ Ovo } \\
\hline & \multicolumn{2}{|c|}{1} & \multicolumn{2}{|c|}{2} & \multicolumn{2}{|c|}{3} & \multicolumn{2}{|c|}{4} & \multicolumn{2}{|c|}{5} & \multicolumn{2}{|c|}{ Média/fêmea } \\
\hline & $\mathrm{C}$ & L & $\mathrm{C}$ & $\mathrm{L}$ & $\mathrm{C}$ & L & $\mathrm{C}$ & $\mathrm{L}$ & $\mathrm{C}$ & L & C & $\mathrm{L}$ \\
\hline 1 & 1,09 & 0,62 & 1,08 & 0,65 & 1,06 & 0,67 & 1,12 & 0,60 & 1,03 & 0,65 & 1,08 & 0,64 \\
\hline 2 & 0,93 & 0,75 & 1,10 & 0,63 & 0,84 & 0,61 & 1,12 & 0,61 & 0,98 & 0,56 & 0,99 & 0,63 \\
\hline 3 & 1,16 & 0,57 & 1,14 & 0,61 & 1,07 & 0,62 & 1,03 & 0,61 & 1,06 & 0,65 & 1,09 & 0,61 \\
\hline 4 & 1,07 & 0,71 & 1,09 & 0,61 & 0,80 & 0,58 & 1,03 & 0,66 & 1,03 & 0,63 & 1,00 & 0,64 \\
\hline 5 & 1,14 & 0,68 & 1,09 & 0,68 & 1,01 & 0,59 & 1,01 & 0,60 & 1,09 & 0,63 & 1,06 & 0,64 \\
\hline 6 & 1,16 & 0,66 & 1,02 & 0,60 & 1,04 & 0,61 & 1,06 & 0,57 & 1,07 & 0,64 & 1,07 & 0,62 \\
\hline 7 & 1,12 & 0,69 & 1,06 & 0,66 & 1,08 & 0,64 & 1,08 & 0,67 & 1,10 & 0,66 & 1,09 & 0,66 \\
\hline 8 & 1,06 & 0,68 & 1,06 & 0,67 & 0,99 & 0,62 & 1,08 & 0,65 & 1,07 & 0,59 & 1,05 & 0,64 \\
\hline 9 & 1,18 & 0,61 & 1,14 & 0,59 & 1,05 & 0,64 & 1,02 & 0,64 & 1,06 & 0,61 & 1,09 & 0,62 \\
\hline 10 & 1,03 & 0,66 & 1,04 & 0,63 & 1,08 & 0,61 & 1,08 & 0,62 & 1,10 & 0,63 & 1,06 & 0,63 \\
\hline 11 & 1,01 & 0,63 & 1,08 & 0,67 & 1,01 & 0,60 & 1,11 & 0,59 & 1,08 & 0,59 & 1,06 & 0,62 \\
\hline 12 & 1,06 & 0,65 & 1,13 & 0,65 & 1,07 & 0,68 & 1,08 & 0,67 & 1,10 & 0,63 & 1,09 & 0,66 \\
\hline 13 & 1,07 & 0,63 & 1,03 & 0,64 & 1,13 & 0,65 & 1,08 & 0,65 & 1,08 & 0,68 & 1,08 & 0,65 \\
\hline 14 & 1,06 & 0,57 & 1,08 & 0,59 & 1,07 & 0,60 & 1,07 & 0,64 & 1,03 & 0,65 & 1,06 & 0,61 \\
\hline 15 & 1,03 & 0,65 & 1,09 & 0,63 & 1,09 & 0,64 & 1,11 & 0,60 & 1,12 & 0,63 & 1,09 & 0,63 \\
\hline 16 & 1,02 & 0,61 & 0,99 & 0,65 & 1,12 & 0,66 & 1,10 & 0,67 & 1,12 & 0,64 & 1,07 & 0,65 \\
\hline 17 & 1,02 & 0,65 & 1,03 & 0,61 & 1,06 & 0,64 & 1,08 & 0,63 & 1,11 & 0,64 & 1,06 & 0,63 \\
\hline 18 & 1,08 & 0,58 & 1,10 & 0,63 & 1,06 & 0,61 & 1,07 & 0,64 & 1,11 & 0,62 & 1,09 & 0,62 \\
\hline 19 & 0,96 & 0,61 & 1,02 & 0,59 & 1,07 & 0,66 & 0,99 & 0,59 & 1,09 & 0,62 & 1,03 & 0,61 \\
\hline 20 & 1,06 & 0,64 & 1,18 & 0,62 & 1,08 & 0,65 & 1,07 & 0,71 & 1,04 & 0,60 & 1,09 & 0,64 \\
\hline Média & - & - & - & - & - & - & - & - & - & - & $1,06 \pm 0,03$ & $0,63 \pm 0,02$ \\
\hline
\end{tabular}

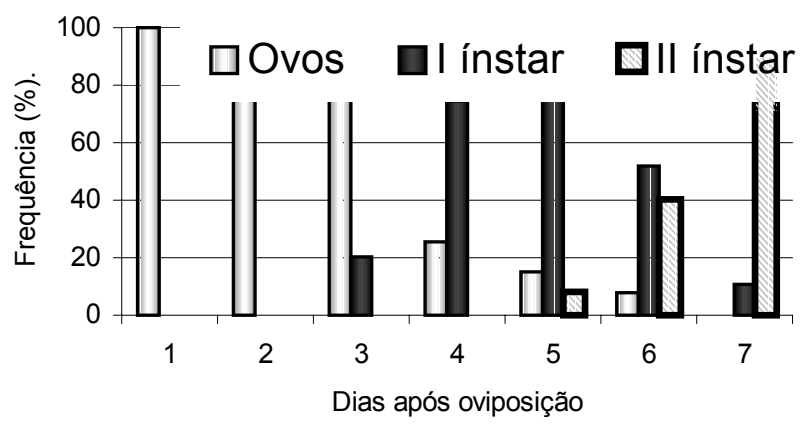

FIGURA 2 - Freqüência da fase de ovo, larva de primeiro e segundo ínstares de Spermologus rufus Boheman ( $\mathrm{T}=24,9 \pm 0,9^{\circ} \mathrm{C}, \mathrm{UR}=78,5 \pm 5,9 \%$ e fotofase $=12$ horas $)$. 

TABELA 2 - Viabilidade (\%) da fase de ovo de Spermologus rufus Boheman ( $\mathrm{T}=24,9 \pm 0,9^{\circ} \mathrm{C}, \quad$ UR $=78,5 \pm 5,9 \%$ e fotofase $=12$ horas), Viçosa, MG, 1997

\begin{tabular}{cccc}
\hline $\begin{array}{c}\text { Fêmea } \\
\text { Número }\end{array}$ & $\begin{array}{c}\text { Viabilidade } \\
(\%)\end{array}$ & $\begin{array}{c}\text { Fêmea } \\
\text { Número }\end{array}$ & $\begin{array}{c}\text { Viabilidade } \\
(\%)\end{array}$ \\
\hline 1 & 97,3 & 12 & 97,6 \\
2 & 99,2 & 13 & 98,1 \\
3 & 95,2 & 14 & 98,7 \\
4 & 99,6 & 15 & 97,6 \\
5 & 98,2 & 16 & 98,7 \\
6 & 97,7 & 17 & 98,6 \\
7 & 98,0 & 18 & 99,5 \\
8 & 98,7 & 19 & 97,2 \\
9 & 98,3 & 20 & 99,3 \\
10 & 91,8 & 21 & 98,5 \\
11 & 97,4 & 22 & 98,7 \\
\hline \multicolumn{4}{c}{}
\end{tabular}

A mortalidade média foi de $2,1 \%$. Destes, $0,2 \%$ foi resultante da presença do ovo no interior da extremidade posterior da semente, o que não permitiu o surgimento de larvas por se tratar de local seco e diferente dos demais tecidos formadores do pinhão [12]; e 1,9\% pode ter ocorrido em função de algumas sementes $(3,5 \%$ da quantidade total de sementes utilizadas), embora ovipositadas, encontrarem-se deterioradas.

Nas observações realizadas nos ovos de $S$. rufus durante o período de incubação foi observado que, no terceiro ou quarto dia, seu conteúdo deslocava-se da periferia para a sua porção central sendo esta a única mudança visível durante o desenvolvimento embrionário, não tendo sido possível visualizar quaisquer estruturas do inseto em formação no interior do ovo.

O fato de o ovo ser depositado no interior da semente pode ser considerado como mecanismo de defesa. Além disso, a coloração do ovo é semelhante à do substrato onde ele é depositado. Esta constatação foi feita por Hinton (1981) [9] e DelClaro (1996) [8] na classe Insecta. Hinton (1981) [9], ao descrever sobre meios de defesa dos ovos, relatou que espécies de lepidópteros das famílias Pyralidae e Olethreutidae ovipositam em locais de coloração semelhante à de seus ovos; dessa forma, durante o seu desenvolvimento, o embrião é protegido da ação externa de inimigos naturais. Além disso, o fato de o ovo ser depositado no interior da semente pode contribuir para o entendimento sobre a dispersão de $S$. rufus, visto que sementes com ovos podem ser disseminadas por perdizes (Rhynchotus rufescens) e por esquilos (Sciurus aestuans) conforme relatos de Sick (1984) [16] e pela gralha-azul (Cyanocorax caeruleus) quando estes procuram tais sementes como fonte de alimento [1] e pelo próprio homem que as comercializa.

\section{REFERÊNCIAS}

[1] ALBERTS, C. C. O esquilo e o pinheiro do Paraná: uma interação. In: CONGRESSO NACIONAL SOBRE ESSÊNCIAS NATIVAS, 2., 1992, São Paulo. Anais. São Paulo: s.n., 1992. Revista do Instituto Florestal, v.4, p. 1215-1216, 1992.

[2] BARRETO, M. R., ANJOS, N., SOUZA, M. P. Ocorrência de Spermologus rufus (Coleoptera: Curculionidae) em sementes de Araucaria angustifolia. Anais da Sociedade Entomológica do Brasil, Londrina, v.25, n.3, p. 567-568, 1996.

[3] BARRETO, M. R. Biologia de Spermologus rufus Boheman (Coleoptera: Curculionidae) em sementes de Araucaria angustifolia (Bert.) Kuntze. Viçosa, 1997, $75 \mathrm{f}$. Dissertação (Mestrado em Entomologia) Universidade Federal de Viçosa.

[4] BONDAR, G. O Cacao II - moléstias e inimigos do cacaoeiro no Estado da Bahia, Brasil. Salvador: Imprensa Official, 1925. $126 \mathrm{p}$.

[5] BORGES, J. D. Biologia de Dirphia araucariae, em laboratório. Pesquisa Agropecuária Brasileira, Brasília, v.20, n.2, p. 155-158, 1985.

[6] BORGES, J. D. Entomofauna do Pinheiro-do-Paraná. Pesquisa Agropecuária Brasileira, Brasília, v.25, n.2, p. 201-206, 1990.

[7] BRASIL. Regras para análise de sementes. Brasília: Ministério da Agricultura e Reforma Agrária, 1992. $365 \mathrm{p}$.

[8] DEL-CLARO, K. A função defensiva da coloração animal. Anais de Etologia, Uberlândia, v.14, p. 175-183, 1996.

[9] HINTON, H. E. Biology of insect eggs. Oxford: Pergamom Press, 1981. $471 \mathrm{p}$

[10] JULIATTO, S. T. Aspectos da biologia de Marshallius bonellii (Boheman, 1830) (Coleoptera: Curculionidae: Hylobiinae) praga da figueira (Ficus carica L.). Piracicaba, 1985, 43 f. Dissertação (Mestrado em Entomologia) - Escola Superior de Agricultura Luiz de Queiroz, Universidade de São Paulo.

[11]LORENZI, H. Árvores brasileiras: manual de identificação e cultivo. São Paulo: Plantarum, 1992. $368 \mathrm{p}$.

[12]MATTOS, J. R. O pinheiro brasileiro. 2.ed. São Paulo: s. n., 1972. $618 \mathrm{p}$.

[13]MOORHOUSE, E. R., CHARNLEY, A. K., GILLESPIE, A. T. A review of the biology and control of the vine weevil, Otiorhynchus sulcatus (Coleoptera: Curculionidae). Annals Applied Biology, Wellessbourne, v.121, p.431-454, 1992.

[14]MOORHOUSE, E. R., FENLON, J. S., GILLESPIE, A. T.; CHARNLEY, A. K. Observations on the development, oviposition and fecundity of Vine Weevil adults, Otiorhynchus sulcatus (Fabricius) (Coleoptera: Curculionidae). The Entomologist's Gazette, Wallingford, v.43, p. 207-218, 1992.

[15]PEREIRA, M. J. B. Biologia de Bephratelloides pomorum (Fab.) (Hymenoptera: Eurytomidae), uma broca da semente da graviola. Viçosa, 1996, $71 \mathrm{f}$. Dissertação (Mestrado em Entomologia) - Universidade Federal de Viçosa.

[16] SICK, H. Ornitologia brasileira, uma introdução. 2.ed. Brasília: Universidade de Brasília, 1984. 952 p.

[17]SILVA, A. G. A., GONÇALVES, C. R., GALVÃO, D. M.; GONÇALVES, A. J. L.; GOMES, J.; SILVA, M. N.; SIMONI, L. Quarto catálogo dos insetos que vivem no Brasil; seus parasitos e predadores. Rio de Janeiro: Ministério da Agricultura, 1968. 622 p.

[18]VIÑUELA, E., ADAN, A., DEL ESTAL, P.; MARCO, V.; BUDIA, F. Plagas de los productos almacenados. Madrid: Unidad de protección de cultivos. E.T.S.I.A. Ministério de Agricultura, Pesca y Alimentacion, 1993. $31 \mathrm{p}$.

Recebido para publicação em 15 JUL 2001 [SA 052/2001] Aceito para publicação em 04 JAN 2002 